09

\title{
Об ускорении релятивистской частицы импульсом излучения
}

\author{
(c) Н.Н. Розанов ${ }^{1,2,3}$ \\ ${ }^{1}$ Государственный оптический институт им. С.И. Вавилова, \\ 199053 Санкт-Петербург, Россия \\ ${ }^{2}$ Университет ИТМО, \\ 197101 Санкт-Петербург, Россия \\ ${ }^{3}$ ФТИ им. А.Ф. Иоффре, \\ 194021 Санкт-Петербург, Россия \\ e-mail: nnrosanov@mail.ru
}

Поступила в редакцию 02.10 .2018 г.

\begin{abstract}
Применительно к прямому ускорению зарядов импульсами электромагнитного (лазерного) излучения для произвольной формы импульса найдено соотношение между компонентами механического импульса релятивистской (но не ультрарелятивистской) частицы. Передаваемая излучением частице энергия в широком диапазоне условий определяется электрической площадью лазерного импульса, т.е. интегралом от напряженности электрического поля по времени. Это указывает на перспективность разработки схем генерации излучения квазиуниполярных лазерных импульсов, у которых напряженность преобладающей компоненты электрического поля не меняет знак в течение основной продолжительности импульса.
\end{abstract}

DOI: $10.21883 /$ OS.2019.02.47206.287-18

\section{Введение}

Прямое ускорение заряженных частиц „обычными“ биполярными - лазерными импульсами считается неэффективным, так как поле излучения на различных оптических периодах то ускоряет, то замедляет заряд. Положение меняется при использовании лазерных пучков с радиальной поляризацией излучения, обладающих заметной продольной составляющей электрической напряженности, см. [1] и многочисленные ссылки в этой работе. В то же время в ряде работ, включая [26], отмечена перспективность прямого лазерного ускорения зарядов униполярными или квазиуниполярными импульсами излучения, поскольку для них знак напряженности основных компонент поля не меняется со временем в течение основной длительности импульса. В нерелятивистском - как классическом, так и квантовом вариантах - воздействие такого импульса на одиночный заряд (электрон) полностью определяется электрической площадью импульса - интегралом от электрической напряженности по времени [7]. Задачей настоящего сообщения служит анализ эффективности прямого лазерного ускорения заряженной частицы в классическом релятивистском режиме, когда скорости заряда становятся значительными, но еще не существенны квантово-электродинамические эффекты и силы радиационного торможения (обсуждение этих эффектов приведено в Заключении).

\section{Исходные уравнения}

Для плоских волн с линейной поляризацией, распространяющихся вдоль $z$, напряженности электрическо- го $\mathbf{E}$ и магнитного Н полей имеют вид

$$
\mathbf{E}=E(z, t) \mathbf{e}_{x}, \quad \mathbf{H}=H(z, t) \mathbf{e}_{y},
$$

где $t-$ время, а $\mathbf{e}_{x}$ и $\mathbf{e}_{e}-$ единичные векторы вдоль осей $x$ и $y$ соответственно. Точные решения уравнений Максвелла, отвечающие однонаправленному распространению, записываются в форме [8]

$$
E(z, t)=H(z, t)=E(z-c t)
$$

при произвольной форме импульса $E(z-c t)$. При этом $c$ - скорость света в вакууме. Далее считаем импульс локализованным, обладающим конечной (на единицу поперечной площади) плотностью энергии.

Релятивистское уравнение движения частицы под действием силы $\mathbf{F}$ совпадает по форме с нерелятивистским вторым законом Ньютона

$$
\frac{d \mathbf{p}}{d t}=\mathbf{F}
$$

Здесь $\mathbf{p}-$ механический импульс частицы с массой $m$. Отличие от нерелятивистской механики состоит в форме связи импульса со скоростью частицы $\mathbf{v}[8]$ :

$$
\mathbf{p}=\frac{m \mathbf{v}}{\sqrt{1-v^{2} / c^{2}}} .
$$

При этом (кинетическая) энергия свободной частицы

$$
E=\frac{m c^{2}}{\sqrt{1-v^{2} / c^{2}}} .
$$

При воздействии на заряд $q$ импульса электромагнитного излучения под $\mathbf{F}$ следует понимать силу Лоренца:

$$
\frac{d \mathbf{p}}{d t}=q \mathbf{E}+\frac{q}{c}[\mathbf{v} \times \mathbf{H}] .
$$


Опущенным аргументом функций $\mathbf{E}$ и $\mathbf{H}$ служит комбинация $z(t)-c t$, где $z(t)-$ продольная координата частицы. Будем считать, что до прихода импульса излучения частица неподвижна и расположена в начале координат $(\mathbf{p}=0$ и $\mathbf{r}=0$ при $t<0 ; \mathbf{r}=(x, y, z)-$ радиус-вектор). Этого всегда можно достичь переходом в соответствующую систему отсчета.

Запись (6) по компонентам ведет к следующим уравнениям:

$$
\frac{d p_{z}}{d t}=\frac{q}{c} E v_{x}, \quad \frac{d p_{x}}{d t}=q E\left(1-\frac{v_{z}}{c}\right), \quad \frac{d p_{y}}{d t}=0 .
$$

С учетом зависимости $E$ от $z$ для замыкания уравнений (7) к ним следует добавить соотношение $\frac{d z}{d t}=v_{z}$.

Из последнего уравнения в (7) с учетом нулевых начальных условий следует, что траектория частицы расположена в плоскости $(x, z)$ :

$$
p_{y}=0, \quad y=0 \text {. }
$$

Теперь, привлекая (4), можно представить (7) в виде системы двух уравнений

$$
\begin{gathered}
\frac{d p_{x}}{d t}=q E-q E \frac{p_{z}}{\sqrt{m^{2} c^{2}+p_{x}^{2}+p_{z}^{2}}}, \\
\frac{d p_{z}}{d t}=q E \frac{p_{x}}{\sqrt{m^{2} c^{2}+p_{x}^{2}+p_{z}^{2}}} .
\end{gathered}
$$

Поделив друг на друга первое и второе уравнения системы (9), найдем

$$
\frac{1}{2} \frac{d p_{x}^{2}}{d p_{z}}=\sqrt{m^{2} c^{2}+p_{x}^{2}+p_{z}^{2}}-p_{z} .
$$

Решением (10), удовлетворяющим нулевым начальным условиям, служит соотношение между ненулевыми компонентами импульса частицы

$$
p_{x}^{2}=2 m c p_{z} .
$$

Важно, что в (10) и (11) не фигурирует профиль импульса излучения. Ввиду этого соотношение (11) служит обобщением на случай произвольных импульсов излучения соотношения, известного для однородного стационарного поля с ортогональными и равными по модулю электрической и магнитной напряженностями [8].

Соотношение (11) позволяет упростить вид динамических уравнений. В частности, связь продольных компонент скорости и импульса записывается в форме

$$
\frac{v_{z}}{c}=\frac{1}{c} \frac{d z}{d t}=\frac{p_{z}}{m c+p_{z}}
$$

а второе из уравнений движения (9) сводится к следующему:

$$
\frac{d p_{z}}{d t}=\sqrt{2 m c} q E(z-c t) \frac{\sqrt{p_{z}}}{m c+p_{z}} .
$$

Отсюда следует соотношение для продольной компоненты импульса частицы

$$
\begin{aligned}
& \left(\frac{p_{z}}{m c}\right)^{1 / 2}+\frac{1}{3}\left(\frac{p_{z}}{m c}\right)^{3 / 2} \\
& \quad=\frac{q}{\sqrt{2} m c} \int_{0}^{t} E\left(-c \int_{0}^{t^{\prime}} \frac{d t^{\prime \prime}}{1+\left[p_{z}\left(t^{\prime \prime}\right) / m c\right]}\right) d t^{\prime} .
\end{aligned}
$$

В частности, после окончания импульса излучения

$$
\begin{aligned}
& \left(\frac{p_{z \infty}}{m c}\right)^{1 / 2}+\frac{1}{3}\left(\frac{p_{z \infty}}{m c}\right)^{3 / 2} \\
& =\frac{q}{\sqrt{2} m c} \int_{0}^{\infty} E\left(-c \int_{0}^{t} \frac{d t^{\prime}}{1+\left[p_{z}\left(t^{\prime}\right) / m c\right]}\right) d t .
\end{aligned}
$$

Для медленного, заведомо нерелятивистского движения $(p=|\mathbf{p}| \ll m c)$ найдем, что конечное состояние частицы полностью определяется электрической площадью импульса $S$ :

$$
S=\int_{-\infty}^{+\infty} E d t
$$

В этом случае знание электрической площади импульса позволяет определить по (15) конечный продольный импульс частицы, по (11) поперечный импульс и соответственно конечную энергию ускоренной заряженной частицы. Заметим, что условие $p=m c$ выполняется при $v=c / \sqrt{2}$, т.е. ниже порога проявления квантовоэлектродинамических эффектов. Однако для релятивистского движения результат зависит в общем случае и от формы импульса излучения, что требует его конкретизации. Здесь следует указать на возможности управлять формой даже предельно коротких импульсов излучения $[3,5,9,10]$.

\section{Импульс излучения прямоугольной формы}

Рассмотрим импульс излучения, для которого на интервале длительности $T$ амплитуда равна постоянной величине $E_{m}$, а вне этого интервала обращается в нуль:

$$
E(z-c t)= \begin{cases}E_{m}, & -c T<z-c t<0, \\ 0, & z-c t<c T, \quad z-c t>0 .\end{cases}
$$

На протяжении времени воздействия импульса на частицу поле можно считать постоянным и однородным. При этом сравнительно простое движение частицы описывается указанным в [8] образом. Согласно (17), на первоначально неподвижную частицу, расположенную в начале координат, импульс излучения начинает воздействовать при $t=0$. Момент окончания воздействия $t^{*}$ определяется условием

$$
z\left(t^{*}\right)=c\left(t^{*}-T\right) .
$$


Опуская выкладки, которые отличаются от приведенных в [8], главным образом, обозначениями и упрощением за счет задания нулевой начальной скорости частицы, приведем простое соотношение для энергии частицы в момент времени $t^{*}$ :

$$
E^{*}=m c^{2}+\frac{(q S)^{2}}{2 m} .
$$

Здесь электрическая площадь импульса $S=E_{m} T$. Естественно, что энергия частицы (как и ее импульс) не меняется в дальнейшие моменты времени.

\section{Заключение}

Таким образом, представлено справедливое для произвольной формы импульса излучения соотношение (11) между компонентами импульса заряженной частицы, ускоряемой импульсом излучения. Оно записано в системе координат, в которой исходная частица (до взаимодействия $\mathrm{c}$ импульсом излучения) покоится, что достаточно, так как более общий случай сводится просто к переходу в другую инерциальную систему координат.

Показано, что электрическая площадь импульса позволяет полностью определить конечные характеристики ускоряемой заряженной частицы в нерелятивистском случае при произвольной форме импульса излучения (для нерелятивистских объектов аналогичный вывод содержится в [7]), а для (классических) релятивистских объектов - при форме импульса излучения, близкой к прямоугольной. В общем случае результат зависит и от самой формы импульса излучения. Это вызвано тем, что во время взаимодействия с частицей непрямоугольного импульса излучения поле в месте нахождения частицы меняется. Воздействие серии лазерных импульсов на заряд описывается последовательным применением полученных результатов с пересчетом напряженности поля при переходе к системе координат, в которой заряд неподвижен после предыдущего импульса.

Область применимости представленного рассмотрения ограничено пренебрежением, во-первых, квантовоэлектродинамическими эффектами и, во-вторых, торможением излучением (лоренцевыми силами трения) [8]. Эти эффекты становятся существенными в ультрарелятивистском режиме, если исходно неподвижный заряд под действием излучения с интенсивностью, превышающей пороговую $I=1.37 \cdot 10^{18} \mathrm{~W} / \mathrm{cm}^{2}$, достигает скоростей, близких к скорости света; соотношение между классическими и квантово-электродинамическими эффектами в таком режиме анализировалось, например, в [11].

Результаты показывают, что эффективность воздействия предельно коротких импульсов на различные микрообъекты в широком диапазоне условий определяется не столько энергией импульса излучения, сколько электрической площадью этого импульса. Поэтому перспективной представляется разработка методов генерации квазиуниполярных импульсов. Такой вывод существен применительно к проектированию сверхмощных лазерных систем „экстремального света“ [12]. Примечательна здесь роль электрической площади, как и в ряде проблем электродинамики сплошных сред [13].

Эта работа поддержана и входит в план исследований по грантам РФФИ 16-02-00762 и 19-02-00312, также по Программе Президиума РАН по нелинейной динамике.

\section{Список литературы}

[1] Carbajo S., Nanni E.A., Liang Jie Wong, Moriena G., Keathley P.D., Laurent G., Dwayne Miller R.J., Kärtner F.X. // Phys. Rev. Accel. Beams. 2016. V. 19. P. 021303. doi 10.1103/PhysRevAccelBeams.19.021303

[2] Song X., Yang $W$., Zeng Z., Li R., Xu Z. // Phys. Rev. A. 2010. V. 82. P. 053821. https://journals.aps.org/ pra/abstract/10.1103/PhysRevA.82.053821

[3] Arkhipov R.M., Pakhomov A.V., Babushkin I.V., Arkhipov M.V., Tolmachev Yu.A., Rosanov N.N. // JOSA B. 2016. V. 33. N 12. P. 2518-2524. https://www.osapublishing.org/ josab/abstract.cfm?uri=josab-33-12-2518

[4] Архипов Р.М., Пахомов А.В., Архипов М.В., Бабушкин И., Толмачев Ю.А., Розанов Н.Н. // Письма в ЖЭТФ. 2017. T. 105. № 6. C. $388-400$. http://www.jetpletters.ac.ru/ps/2151/article_32278.shtml

[5] Pakhomov A.V., Arkhipov R.M., Babushkin I.V., Arkhipov M.V., Tolmachev Yu.A., Rosanov N.N. // Phys. Rev. A. 2017. V. 95. N 1. P. 013804. https://journals.aps.org/ pra/abstract/10.1103/PhysRevA.95.013804

[6] Ziguleva D.O., Arkhipov R.M., Arkhipov M.V., Pakhomov A.V., Babushkin I., Rosanov N.N. // Opt. Commun. 2018. V. 424. P. 170-176. https://www.sciencedirect.com/science/ article/abs/pii/S0030401818303195

[7] Розанов Н.Н. // Опт. и спектр. 2018. Т. 125. № 6. С. 818.

[8] Ландау Л.Л., Лифиии, Е.М. Теория поля. М.: Наука, 1988. $512 \mathrm{c}$.

[9] Weiner A.M. // Opt. Commun. 2011. V. 284. N 15. P. 3669-3692.

[10] Arkhipov R.M., Pakhomov A.V., Arkhipov M.V., Babushkin I., Tolmachev Yu.A., Rosanov N.N. // Laser Physics. 2017. V. 27. N 5. Р. 053001-10. Электронный pecypc. Режим доступа: http://iopscience.iop.org/article/10.1088/15556611/aa64b6/meta

[11] Esirkepov T.Zh., Bulanov S.S., Koga J.K., Kando M., Kondo K., Rosanov N.N., Korn G., Bulanov S.V. // Phys. Lett. A. 2015. V. 379. N 36. P. 2044-2054. doi 10.1016/j.physleta.2015.06.017

[12] Электронный ресурс. Режим доступа: https://eli-laser.eu/

[13] Розанов Н.Н., Архипов М.В., Архипов Р.М. // УФН. 2018. doi 10.3367/UFNr.2018.07.038386; Rosanov N.N., Arkhipov M.V., Arkhipov R.M. // Phys. Usp. 2018. doi 10.3367/UFNe.2018.07.038386 